\title{
Divergence and Curl Operators in Skew Coordinates
}

\author{
Rajai S. Alassar and Mohammed A. Abushoshah
}

\begin{abstract}
The divergence and curl operators appear in numerous differential equations governing engineering and physics problems. These operators, whose forms are well known in general orthogonal coordinates systems, assume different casts in different systems. In certain instances, one needs to custom-make a coordinates system that my turn out to be skew (i.e. not orthogonal). Of course, the known formulas for the divergence and curl operators in orthogonal coordinates are not useful in such cases, and one needs to derive their counterparts in skew systems. In this note, we derive two formulas for the divergence and curl operators in a general coordinates system, whether orthogonal or not. These formulas generalize the well known and widely used relations for orthogonal coordinates systems. In the process, we define an orthogonality indicator whose value ranges between zero and unity.
\end{abstract}

Index Terms-Coordinates systems, curl, divergence, Laplace, skew systems.

\section{INTRODUCTION}

The curl and divergence operators play significant roles in physical relations. They arise in fluid mechanics, elasticity theory and are fundamental in the theory of electromagnetism, [1], [2].

The physical significance of the Curl of a vector field $\vec{F}$, denoted by $\vec{\nabla} \times \vec{F}$, is that it measures the amount of rotation or angular momentum of the contents of a given region of space. If the value of the curl is zero then the field is said to be irrotational. The curl is defined in an arbitrary orthogonal curvilinear coordinates $\left(u_{1}, u_{2}, u_{3}\right)$ as

$$
\vec{\nabla} \times \vec{F}=\frac{1}{h_{1} h_{2} h_{3}}\left|\begin{array}{ccc}
h_{1} \boldsymbol{e}_{1} & h_{2} \boldsymbol{e}_{2} & h_{3} \boldsymbol{e}_{3} \\
\frac{\partial}{\partial u_{1}} & \frac{\partial}{\partial u_{2}} & \frac{\partial}{\partial u_{3}} \\
F_{1} h_{1} & F_{2} h_{2} & F_{3} h_{3}
\end{array}\right|
$$

where $\boldsymbol{e}_{i}$ is a unit vector in the direction of $u_{i}$, and $\vec{F}=F_{1} e_{1}+F_{2} e_{2}+F_{3} e_{3}$. The length of the tangent vector in the direction of $u_{i}$ is known as scale factor, $h_{i}$, and is defined by

$$
h_{i}=\left|\frac{\partial \vec{r}}{\partial u_{i}}\right|
$$

Manuscript received March 29, 2015; revised May 19, 2015. This work was supported by King Fahd University of Petroleum \& Minerals.

The authors are with the Department of Math and Stat, King Fahd University of Petroleum \& Minerals, Dhahran, Saudi Arabia (e-mail: alassar@kfupm.edu.sa.sa, abushosha@kfupm.edu.sa). where $\vec{r}$ is the position vector in any three dimensional space, i.e. $\vec{r}=\vec{r}\left(u_{1}, u_{2}, u_{3}\right)$.

Note that $\frac{\partial \vec{r}}{\partial u_{i}}$ is a tangent vector to the $u_{i}$ curve where the other two coordinates variables remain constant. A unit tangent vector in this direction, therefore, is

$$
\boldsymbol{e}_{i}=\frac{\frac{\partial \vec{r}}{\partial u_{i}}}{\left|\frac{\partial \vec{r}}{\partial u_{i}}\right|}=\frac{\frac{\partial \vec{r}}{\partial u_{i}}}{h_{i}} \quad \text { or } \quad \frac{\partial \vec{r}}{\partial u_{i}}=h_{i} \boldsymbol{e}_{i}
$$

The Divergence of a vector field over a control volume $V$ bounded by the surface $S$, denoted by $\vec{\nabla} \cdot \vec{F}$, is defined by

$$
\vec{\nabla} \cdot \vec{F}=\lim _{V \rightarrow 0} \frac{\oint_{S} \vec{F} \cdot \vec{n} d S}{V}
$$

where $\vec{n}$ is an outward unit normal vector to the surface $S$. The divergence actually measures the net outflow of a vector field from an infinitesimal volume around a given point (or how much a vector field "converges to" or "diverges from" a given point). The general expression of the divergence for arbitrary orthogonal curvilinear coordinates is given by

$$
\begin{aligned}
\vec{\nabla} \cdot \vec{F}=\frac{1}{h_{1} h_{2} h_{3}}[ & \frac{\partial}{\partial u_{1}}\left(F_{1} h_{2} h_{3}\right)+ \\
& \left.\frac{\partial}{\partial u_{2}}\left(F_{2} h_{1} h_{3}\right)+\frac{\partial}{\partial u_{3}}\left(F_{3} h_{1} h_{2}\right)\right]
\end{aligned}
$$

The Laplacian operator of a scalar $\psi=\psi\left(u_{1}, u_{2}, u_{3}\right)$, denoted by $\nabla^{2} \psi$ is defined as the divergence of the gradient of $\psi$; that is

$$
\begin{array}{r}
\nabla^{2} \psi=\vec{\nabla} \cdot(\vec{\nabla} \psi)=\frac{1}{h_{1} h_{2} h_{3}}\left[\frac{\partial}{\partial u_{1}}\left(\frac{h_{2} h_{3}}{h_{1}} \frac{\partial \psi}{\partial u_{1}}\right)+\right. \\
\left.\frac{\partial}{\partial u_{2}}\left(\frac{h_{1} h_{3}}{h_{2}} \frac{\partial \psi}{\partial u_{2}}\right)+\frac{\partial}{\partial u_{3}}\left(\frac{h_{1} h_{2}}{h_{3}} \frac{\partial \psi}{\partial u_{3}}\right)\right]
\end{array}
$$

where

$$
\vec{\nabla} \psi=\frac{1}{h_{1}} \frac{\partial \psi}{\partial u_{1}} \boldsymbol{e}_{1}+\frac{1}{h_{2}} \frac{\partial \psi}{\partial u_{2}} \boldsymbol{e}_{2}+\frac{1}{h_{3}} \frac{\partial \psi}{\partial u_{3}} \boldsymbol{e}_{3}
$$

Equations (1) and (5) are valid for orthogonal systems 
only (i.e. $\boldsymbol{e}_{1}, \boldsymbol{e}_{2}$, and $\boldsymbol{e}_{3}$ are orthogonal), [3]-[5].

\section{DiVERGENCE FOR GENERAL COORDINATES SYSTEMS}

The Divergence Theorem relates the flow, or the flux, of a vector field through a surface to the behavior of the vector field inside the surface. More precisely, it states that the outward flux of a vector field through a closed surface is equal to the volume integral of the divergence over the region inside the surface, i.e.

$$
\iint_{S} \vec{w} \bullet \vec{n} d S=\iiint_{D} \vec{\nabla} \bullet \vec{w} d v
$$

where $D$ is a closed bounded region with piecewise smooth boundary $S, \vec{n}$ is an outer unit vector normal to the surface $S, \vec{w}=w_{1} \boldsymbol{e}_{1}+w_{2} \boldsymbol{e}_{2}+w_{3} \boldsymbol{e}_{3}, \vec{w} \cdot \vec{n}$ represents the component of $\vec{w}$ in the direction of $\vec{n}$, and $d v$ is the volume bounded by the region $D$.

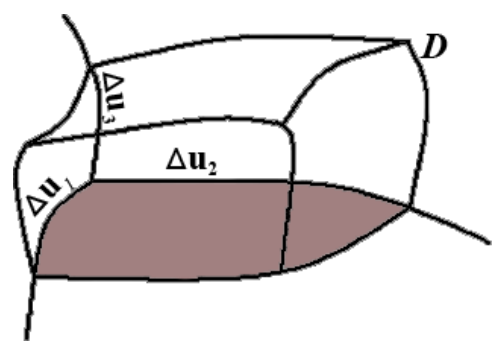

Fig. 1. An infinitesimal control volume bounded by the surface.

Considering the bottom shaded surface $d S$ of the given control volume, Fig. 1, one can find that

$$
\vec{n}=-\frac{\frac{\partial \vec{r}}{\partial u_{1}} \times \frac{\partial \vec{r}}{\partial u_{2}}}{\left|\frac{\partial \vec{r}}{\partial u_{1}} \times \frac{\partial \vec{r}}{\partial u_{2}}\right|}, \text { and } d S=\left|\frac{\partial \vec{r}}{\partial u_{1}} \times \frac{\partial \vec{r}}{\partial u_{2}}\right| d u_{1} d u_{2}
$$

Hence

$$
\begin{aligned}
\vec{w} \cdot \vec{n} d S=-\vec{w} \bullet \frac{\frac{\partial \vec{r}}{\partial u_{1}} \times \frac{\partial \vec{r}}{\partial u_{2}}}{\left|\frac{\partial \vec{r}}{\partial u_{1}} \times \frac{\partial \vec{r}}{\partial u_{2}}\right|}\left|\frac{\partial \vec{r}}{\partial u_{1}} \times \frac{\partial \vec{r}}{\partial u_{2}}\right| d u_{1} d u_{2} \\
=-\left(w_{1} \boldsymbol{e}_{\mathbf{1}}+w_{2} \boldsymbol{e}_{2}+w_{3} \boldsymbol{e}_{\mathbf{3}}\right) \cdot\left(h_{1} \boldsymbol{e}_{\mathbf{1}} \times h_{2} \boldsymbol{e}_{2}\right) d u_{1} d u_{2} \\
=-w_{3}\left(\boldsymbol{e}_{\mathbf{3}} \bullet \boldsymbol{e}_{\mathbf{1}} \times \boldsymbol{e}_{2}\right) h_{1} h_{2} d u_{1} d u_{2}
\end{aligned}
$$

On the upper surface, using Taylor series, the outward flux is

$$
\begin{aligned}
& w_{3}\left(\boldsymbol{e}_{3} \bullet \boldsymbol{e}_{\mathbf{1}} \times \boldsymbol{e}_{2}\right) h_{1} h_{2} d u_{1} d u_{2}+ \\
& \frac{\partial}{\partial u_{3}}\left[w_{3}\left(\boldsymbol{e}_{3} \bullet \boldsymbol{e}_{1} \times \boldsymbol{e}_{2}\right) h_{1} h_{2}\right] d u_{1} d u_{2} d u_{3}+\ldots
\end{aligned}
$$

The net flux through the upper and lower surfaces, then, is

$$
\frac{\partial}{\partial u_{3}}\left[\left(\boldsymbol{e}_{3} \cdot \boldsymbol{e}_{1} \times e_{2}\right) w_{3} h_{1} h_{2}\right] d u_{1} d u_{2} d u_{3}+\cdots
$$

Using the same argument, the net flux through the remaining two pairs of surfaces are:

$$
\begin{aligned}
& \frac{\partial}{\partial u_{2}}\left[\left(\boldsymbol{e}_{\mathbf{3}} \cdot \boldsymbol{e}_{\mathbf{1}} \times e_{2}\right) w_{2} h_{1} h_{3}\right] d u_{1} d u_{2} d u_{3}+\cdots \\
& \frac{\partial}{\partial u_{1}}\left[\left(\boldsymbol{e}_{\mathbf{3}} \cdot \boldsymbol{e}_{\mathbf{1}} \times e_{2}\right) w_{1} h_{2} h_{3}\right] d u_{1} d u_{2} d u_{3}+\cdots
\end{aligned}
$$

$\vec{w} \bullet \vec{n} d S$ on the left hand side of equation (8) becomes

$$
\begin{aligned}
\left(\frac{\partial}{\partial u_{3}}\left[e w_{3} h_{1} h_{2}\right]+\frac{\partial}{\partial u_{2}}\left[e w_{2} h_{1} h_{3}\right]\right. & \left.+\frac{\partial}{\partial u_{1}}\left[e w_{1} h_{2} h_{3}\right]\right) \\
& \times d u_{1} d u_{2} d u_{3}+\cdots
\end{aligned}
$$

where $e=\boldsymbol{e}_{3} \cdot \boldsymbol{e}_{1} \times e_{2}$ is an orthognality indicator of the system, $(0<e \leq 1)$.

The volume element, $d v$, is given by

$$
d v=\left(h_{3} \boldsymbol{e}_{3} d u_{3}\right) \cdot\left(h_{1} \boldsymbol{e}_{1} d u_{1} \times h_{2} \boldsymbol{e}_{2} d u_{2}\right)
$$

The right hand side of equation (8), then, is

$$
\begin{aligned}
\vec{\nabla} \cdot \vec{w} d v & =\vec{\nabla} \cdot \vec{w}\left(h_{3} \boldsymbol{e}_{3} d u_{3}\right) \cdot\left(h_{1} \boldsymbol{e}_{1} d u_{1} \times h_{2} \boldsymbol{e}_{2} d u_{2}\right) \\
& =\vec{\nabla} \cdot \vec{w}\left(\boldsymbol{e}_{3} \cdot \boldsymbol{e}_{1} \times e_{2}\right)\left(h_{1} h_{2} h_{3} d u_{1} d u_{2} d u_{3}\right) \\
& =\vec{\nabla} \cdot \vec{w} e\left(h_{1} h_{2} h_{3} d u_{1} d u_{2} d u_{3}\right)
\end{aligned}
$$

In the limit, by equating (12) and (14), an expression for the divergence of a vector field $\vec{w}$ in a general coordinates system, whether orthogonal or not, is given by

$$
\begin{aligned}
\vec{\nabla} \cdot \vec{w}=\frac{1}{e h_{1} h_{2} h_{3}}[ & \frac{\partial}{\partial u_{1}}\left(e w_{1} h_{2} h_{3}\right)+ \\
& \left.\frac{\partial}{\partial u_{2}}\left(e w_{2} h_{1} h_{3}\right)+\frac{\partial}{\partial u_{3}}\left(e w_{3} h_{1} h_{2}\right)\right]
\end{aligned}
$$

It is easy to see that when $e=1$ (orthogonal system), equation (15) reduces to (5).

\section{CURL FOR GENERAL COORDINATES SYSTEMS}

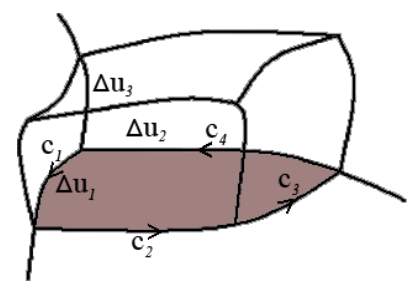

Fig. 2. An infinitesimal surface enclosed by the closed path c. 
Stokes' Theorem relates the surface integral of the curl of a vector field over a surface to the line integral of the vector field over the surface boundary, or

$$
\iint_{S} \vec{\nabla} \times \vec{w} \bullet \vec{n} d S=\oint_{c} \vec{w} \bullet d \vec{r}
$$

Considering the bottom shaded surface of the given control volume as shown in Fig. 2,

This surface is bounded by the curve $c$ which is traced counterclockwise. This curve is comprised of the four segments $c_{1}, c_{2}, c_{3}$, and $c_{4}$. We evaluate the right hand side and the left hand side of (16) over this surface and two other surfaces not opposite to each other. On the shown shaded surface, one can write

$$
\begin{aligned}
\vec{\nabla} \times \vec{w} \cdot \vec{n} d S & = \\
\left((\vec{\nabla} \times \vec{w})_{1} \boldsymbol{e}_{1}+(\vec{\nabla} \times \vec{w})_{2} \boldsymbol{e}_{2}+(\vec{\nabla} \times \vec{w})_{3} \boldsymbol{e}_{3}\right) \cdot & \left(\frac{h_{1} \boldsymbol{e}_{1} \times h_{2} \boldsymbol{e}_{2}}{\left|h_{1} \boldsymbol{e}_{1} \times h_{2} \boldsymbol{e}_{2}\right|}\right)\left|h_{1} \boldsymbol{e}_{\mathbf{1}} \times h_{2} \boldsymbol{e}_{2}\right| d u_{1} d u_{2} \\
= & \left((\vec{\nabla} \times \vec{w})_{3} \boldsymbol{e}_{3}\right) \cdot\left(\boldsymbol{e}_{1} \times \boldsymbol{e}_{2}\right) h_{1} h_{2} d u_{1} d u_{2} \\
= & (\vec{\nabla} \times \vec{w})_{3} e h_{1} h_{2} d u_{1} d u_{2}
\end{aligned}
$$

where $(\vec{\nabla} \times \vec{w})_{i}$ is the component of $\vec{\nabla} \times \vec{w}$ in the direction of $\boldsymbol{e}_{\boldsymbol{i}}$.

The term $\vec{w} \bullet d r$ in equation (16), over the four segments comprising the curve $c$, is

$$
\begin{aligned}
\vec{w} \cdot d \vec{r} & =\left.\vec{w} \cdot d \vec{r}\right|_{c_{1}}+\left.\vec{w} \bullet d \vec{r}\right|_{c_{2}}+\left.\vec{w} \bullet d \vec{r}\right|_{c_{3}}+\left.\vec{w} \bullet d \vec{r}\right|_{c_{4}} \\
& =\left.\vec{w} \cdot d \vec{r}\right|_{c_{1}}+\left.\vec{w} \bullet d \vec{r}\right|_{c_{3}}+\left.\vec{w} \bullet d \vec{r}\right|_{c_{4}}+\left.\vec{w} \bullet d \vec{r}\right|_{c_{2}} \\
& =\left(\vec{w} \cdot h_{1} \boldsymbol{e}_{1} d u_{1}\right)+ \\
& \left(-\vec{w} \cdot h_{1} \boldsymbol{e}_{1} d u_{1}+\frac{\partial}{\partial u_{2}}\left[-\vec{w} \bullet h_{1} \boldsymbol{e}_{1} d u_{1}\right] d u_{2}\right)+\cdots+ \\
& \left(-\vec{w} \cdot h_{2} \boldsymbol{e}_{2} d u_{2}\right)+ \\
& \left(\vec{w} \bullet h_{2} \boldsymbol{e}_{2} d u_{2}+\frac{\partial}{\partial u_{1}}\left[\vec{w} \cdot h_{2} \boldsymbol{e}_{2} d u_{2}\right] d u_{1}\right)+\cdots
\end{aligned}
$$

Or

$$
\vec{w} \bullet d r=\left(\frac{\partial}{\partial u_{1}}\left[\vec{w} \bullet h_{2} \boldsymbol{e}_{2}\right]-\frac{\partial}{\partial u_{2}}\left[\vec{w} \bullet h_{1} \boldsymbol{e}_{1}\right]\right) d u_{1} d u_{2}+\cdots
$$

In the limit, after equating (17) and (18),

$$
(\vec{\nabla} \times \vec{w})_{3}=\frac{1}{e h_{1} h_{2}}\left(\frac{\partial}{\partial u_{1}}\left[h_{2} \vec{w} \bullet e_{2}\right]-\frac{\partial}{\partial u_{2}}\left[h_{1} \vec{w} \bullet e_{1}\right]\right)
$$

Similar results can be obtained for the other two surfaces.

$$
(\vec{\nabla} \times \vec{w})_{2}=\frac{1}{e h_{3} h_{1}}\left(\frac{\partial}{\partial u_{3}}\left[h_{1} \vec{w} \bullet e_{1}\right]-\frac{\partial}{\partial u_{1}}\left[h_{3} \vec{w} \bullet e_{3}\right]\right)
$$

$$
(\vec{\nabla} \times \vec{w})_{1}=\frac{1}{e h_{3} h_{2}}\left(\frac{\partial}{\partial u_{2}}\left[h_{3} \vec{w} \bullet e_{3}\right]-\frac{\partial}{\partial u_{3}}\left[h_{2} \vec{w} \bullet e_{2}\right]\right)
$$

Equations (19), (20) and (21) can be written in the concise form

$$
\vec{\nabla} \times \vec{w}=\frac{1}{e h_{1} h_{2} h_{3}}\left|\begin{array}{ccc}
h_{1} \boldsymbol{e}_{1} & h_{2} \boldsymbol{e}_{2} & h_{3} \boldsymbol{e}_{3} \\
\frac{\partial}{\partial u_{1}} & \frac{\partial}{\partial u_{2}} & \frac{\partial}{\partial u_{3}} \\
h_{1} \vec{w} \bullet e_{1} & h_{2} \vec{w} \bullet e_{2} & h_{3} \vec{w} \bullet e_{3}
\end{array}\right|
$$

Obviously, the difference between orthogonal and nonorthogonal coordinates systems lies in the introduction of the scalar triple product $\boldsymbol{e}_{\mathbf{3}} \boldsymbol{e}_{\mathbf{3}} \cdot \boldsymbol{e}_{\mathbf{1}} \times \boldsymbol{e}_{2}$ into the expressions of $\vec{\nabla} \times \vec{F}$, and $\vec{\nabla} \cdot \vec{F}$. This product returns a value of unity if the coordinates system is orthogonal.

\section{EXAMPLE}

Consider the simple right-handed coordinates system $(u, v, z)$ which is related to the Cartesian system by the relations

$$
\begin{aligned}
& x=u-v \\
& y=u \\
& z=z
\end{aligned}
$$

This system is composed of the planes:

$y=u$ which are planes parallel to the $x-z$ plane, $y=x+v$ which are planes that are extrusions of the straight lines $y=x+u$ into the $z$-direction, and planes that are parallel to the $x-y$ plane.

The level curves of the coordinates system on the $x-y$ plane are shown in Fig. 3.

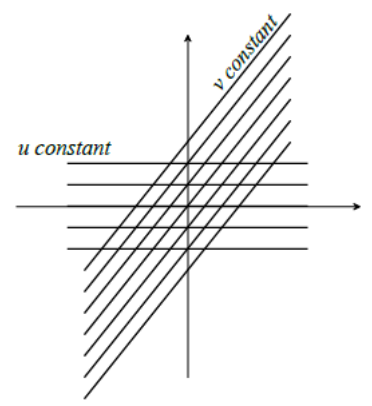

Fig. 3. Example of a non-orthogonal coordinates system.

Obviously, the coordinates system is not orthogonal. Simple calculations reveal that

$$
\begin{aligned}
& h_{1}=h_{u}=\left|\frac{\partial \vec{r}}{\partial u}\right|=\sqrt{2} \\
& h_{2}=h_{v}=\left|\frac{\partial \vec{r}}{\partial v}\right|=1 \\
& h_{3}=h_{z}=\left|\frac{\partial \vec{r}}{\partial z}\right|=1
\end{aligned}
$$


The corresponding unit vectors are

$$
\begin{aligned}
& \boldsymbol{e}_{1}=\frac{\partial \vec{r}}{\partial u} /\left|\frac{\partial \vec{r}}{\partial u}\right|=(i+j) / \sqrt{2} \\
& \boldsymbol{e}_{2}=\frac{\partial \vec{r}}{\partial v} /\left|\frac{\partial \vec{r}}{\partial v}\right|=-i \\
& \boldsymbol{e}_{3}=\frac{\partial \vec{r}}{\partial z} /\left|\frac{\partial \vec{r}}{\partial z}\right|=k
\end{aligned}
$$

Now, the orthogonality indicator is

$$
e=\boldsymbol{e}_{3} \cdot \boldsymbol{e}_{1} \times e_{2}=\left|\begin{array}{ccc}
0 & 0 & 1 \\
\frac{1}{\sqrt{2}} & \frac{1}{\sqrt{2}} & 0 \\
-1 & 0 & 0
\end{array}\right|=\frac{1}{\sqrt{2}}
$$

The value of the orthogonality indicator being different from unity indicates that the system is indeed skew.

One can now find the corresponding operator (15) as

$$
\vec{\nabla} \cdot \vec{F}=\frac{1}{\sqrt{2}} \frac{\partial F_{1}}{\partial u_{1}}+\frac{\partial F_{2}}{\partial u_{2}}+\frac{\partial F_{3}}{\partial u_{3}}
$$

and the three components of the curl in (22) to be

$$
\begin{gathered}
(\vec{\nabla} \times \vec{w})_{1}=\sqrt{2}\left(\frac{\partial w_{3}}{\partial v}-\frac{\partial\left(w_{2}-w_{1} / \sqrt{2}\right)}{\partial z}\right) \\
(\vec{\nabla} \times \vec{w})_{2}=\sqrt{2} \frac{\partial\left(w_{1}-w_{2} / \sqrt{2}\right)}{\partial z}-\frac{\partial w_{3}}{\partial u} \\
(\vec{\nabla} \times \vec{w})_{3}=\frac{\partial\left(-w_{1} / \sqrt{2}+w_{2}\right)}{\partial u}-\sqrt{2} \frac{\partial\left(w_{1}-w_{2} / \sqrt{2}\right)}{\partial v}
\end{gathered}
$$

\section{CONCLUSION}

Two formulas for the divergence and curl operators in any coordinates systems whether orthogonal or not have been obtained. These formulas generalize the well known and widely used relations for orthogonal coordinates systems. The difference between orthogonal and nonorthogonal coordinates systems lies in the introduction of the orthogonality indicator $\boldsymbol{e}_{\mathbf{3}} \boldsymbol{e}_{\mathbf{3}} \boldsymbol{e}_{\mathbf{1}} \times \boldsymbol{e}_{2}$ which returns a value of unity if the coordinates system is orthogonal.

\section{ACKNOWLEDGMENT}

The authors would like to thank King Fahd University of Petroleum \& Minerals (KFUPM) for supporting this research under grant IN131060.

\section{REFERENCES}

[1] R. S. Alassar and H. M. Badr, "Analytical solution of oscillating inviscid flow over oblate spheroids with spheres and flat disks as special cases," Ocean Engng, vol. 24, no. 3, pp. 217-225, 1997.

[2] R. S. Alassar and B. J. Alminshawy, "Heat conduction from two spheres," Aiche Journal, vol. 56, no. 9, pp. 2248-2256, 2010.

[3] O. Moon and D. Spencer, Field Theory for Engineers (The Van Nostrand Series in Electronics and Communications), D. Van Nostrand Co., Inc., Princeton, N.J.-Toronto-London-New York, 1961 pp. $1-48$.

[4] G. Arfken, Mathematical Methods for Physicists, 3rd ed. Orlando, FL: Academic Press, 1985, pp. 100-152.

[5] P. Morse and H. Feshbach, Methods of Theoretical Physics, Part I, New York: McGraw-Hill, 1953, pp. 660-690.

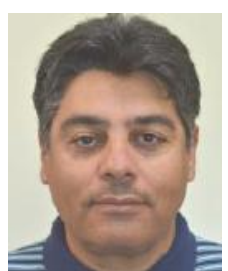

Rajai S. Alassar is a professor in the Department of Mathematics and Statistics at King Fahd University of Petroleum \& Minerals (KFUPM). He was born in Gaza in 1965 . He holds a PhD in hydrodynamics.

He has published many papers in his field of interest and has authored 2 textbooks in Arabic on the subject of Math Olympiad. He has also translated the famous book of Zill on differential equations to Arabic.

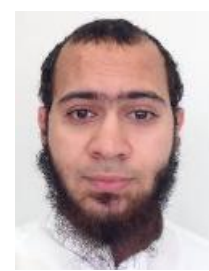

Mohammed Abushoshah is a lecturer in the Department of Mathematics and Statistics at King Fahd University of Petroleum \& Minerals (KFUPM). He was born in Jeddah in 1987. He holds an MSc in applied mathematics.

$\mathrm{He}$ has published several papers in applied mathematics with concentration on fluid mechanics and thermal sciences. 